\title{
Nutritional Status of Centenarians Assessed by Activity and Anthropometric, Hematological and Biochemical Characteristics
}

\author{
Yin-Ching Chan,* Makoto Suzuki, and Shigeru Yamamoto \\ Research Center of Comprehensive Medicine, Faculty of Medicine, \\ University of the Ryukyus, Okinawa 903-01, Japan
}

(Received August 1, 1996)

Summary For the maintenance of good health of very old people such as centenarians, nutrition is very important. However, the information on their nutritional status is very limited. In this study, we evaluated the nutritional status of centenarians by comparison with elderly in their $70 \mathrm{~s}$ and young adults. Volunteer subjects were comprised of 71 centenarians ( 51 females, 20 males) who were not bed ridden, 84 elderly in their $70 \mathrm{~s}$ ( 51 females, 33 males) who had no specific health problems and 27 healthy young adults in their $20 \mathrm{~s}$ (17 females, 10 males). We compared 11 items of the activities of daily living (ADL), height, weight, hematological (hemoglobin, hematocrit and red blood cell count) and biochemical [total protein, albumin, total-, HDL-, LDL- and VLDL-cholesterols, triglycerides, glutamate oxaloacetate transaminase (GOT), glutamate pyruvate transaminase (GPT) and creatinine] variables among the three age groups. Since the atrophy of muscle is more severe than the atrophies of other tissues in the elderly, we studied the muscle protein breakdown using plasma 3-methylhistidine (3-MH) as a marker. The results of all indices except serum lipids, GOT and GPT, were lower in the centenarians than in the subjects in their $20 \mathrm{~s}(p<0.05)$ and were lower than the lower reference limits. Most of the results of the elderly in their $70 \mathrm{~s}$ were within the reference intervals. However, as compared to the young subjects, they had: 1) normal ADL; 2) shorter height; 3) heavier weight in females and lighter weight in males; 4) lower total protein and albumin levels and albumin/globulin (A/G) ratios; and 5) higher total- and LDLcholesterol levels in females and HDL-cholesterol levels in males $(p<$ 0.05). The plasma 3-MH concentration was higher in the centenarian group than in the young and elderly groups $(p<0.05)$. However, serum creatinine concentrations also tended to be high in the group, suggesting that a high 3-MH concentration did not indicate accelerated muscle protein breakdown but rather poor kidney functions. The results of this

\footnotetext{
* To whom correspondence should be addressed.
} 
study show that the centenarians had shrunken stature, low hematological values and poor protein nutrition but rather normal lipid nutrition, however the elderly subjects in their 70 s could still maintain most nutritional parameters in relatively normal condition.

Key Words centenarian, elderly, nutrition, plasma, activity, biochemical values, 3-methylhistidine

The proportion and number of elderly people have progressively increased in most developed countries. Elderly people usually have various health problems. To ensure an optimum quality of life and also alleviate the financial and psychological burdens for elderly people, their families and society as a whole, the maintenance of their health is important. Nutrition is the basis for this. Therefore, information about the nutritional status of elderly people is important. Kawai and his project team (1) have well reviewed the reference intervals of Japanese elderly in the clinical laboratory. The information is very useful; however, information on very old people such as centenarians is not available.

The activities of daily living (ADL), anthropometric variables and hematological and biochemical variables such as hemoglobin $(\mathrm{Hb})$, total protein, albumin, globulin, albumin/globulin $(\mathrm{A} / \mathrm{G})$ ratio and various lipoprotein cholesterols are good indicators of nutritional status. In the elderly, loss of muscle mass is much greater than other organs (2). Therefore, it is important to prevent the loss of muscle mass with aging. In the human body, most 3-methylhistidine (3-MH) is in the muscle, and after the breakdown of muscle protein it is not reutilized. Therefore, it can be a good indicator of muscle protein breakdown $(3,4)$. Muscle protein breakdown is usually calculated by the urinary excretion of 3-MH. Recently, Nagasawa (5) reported that plasma 3-MH concentration is good enough for the comparison of muscle protein breakdown. Collection of $24 \mathrm{~h}$ urine is not easy, especially for the elderly. Therefore, the measurement of plasma 3-MH is more practical for the comparison of muscle mass breakdown among different age groups.

With the above background information, in this study, we tried to assess the nutritional status by ADL and anthropometric, hematological and biochemical variables, and muscle protein breakdown by the plasma 3-MH concentrations of centenarians and subjects in their 20 s and 70 s.

\section{MATERIALS AND METHODS}

The subjects in this study were 182 adult volunteers including 27 young bank workers (10 males aged $26.3 \pm 0.8 \mathrm{y} ; 17$ females aged $25.0 \pm 0.6 \mathrm{y}$ ), 84 healthy elderly ( 33 males aged $74.5 \pm 0.7 y$; 51 females aged $74.7 \pm 0.6 y$ ) and 71 centenarians (20 males aged $100.7 \pm 0.3 \mathrm{y} ; 51$ females aged $100.8 \pm 0.3 \mathrm{y}$ ). The first two groups were citizens who went to the annual health examination of the prefecture 
and agreed to participate in this study. The centenarians were volunteers from the general public. The purpose and procedures of the study were explained to the subjects and also to the families of centenarians, and written consent was obtained from all members. All subjects underwent a physical examination and blood tests (including liver and kidney functions), and those who were taking medication or had chronic illness were excluded from this study. We also measured height and weight.

ADL were assessed by interviewing the subjects and the families of the centenarians. Physical activities (meal taking, bowel and bladder continence, standing ability, extent of general activities, bathing and dressing ability), sensory functions (auditory acuity and eyesight) and cognitive abilities (comprehension and self-expression) were included in the ADL. Each item was classified into five categories of self sufficiency: completely independent, independent but slow, independent with difficulty, partially dependent and completely dependent and scored using a point scale from 5 to 1 , respectively (7).

Blood was withdrawn from the antecubital vein early in the morning (after overnight fasting) and was used for the measurement of hemoglobin ( $\mathrm{Hb})$, hematocrit (Ht) and red blood cell count (RBC). A part of the blood was centrifuged $(3,000 \mathrm{rpm}$ for $15 \mathrm{~min})$ to separate serum for analysis of total protein, albumin, total-cholesterol, HDL-cholesterol, LDL-cholesterol and VLDL-cholesterol, triglycerides, glutamate oxaloacetate transaminase (GOT), glutamate pyruvate transaminase (GPT) and creatinine. The hematological and biochemical parameters were analyzed at a commercial analytical service center (SRL Co., Okinawa, Japan). The globulin concentration was obtained by subtracting albumin concentration from total protein concentration, and the albumin/globulin $(\mathrm{A} / \mathrm{G})$ ratio was calculated.

For 3-MH determination, a part of the whole blood was dispensed into a heparinized tube and plasma was obtained after centrifugation, and then stored at $-80^{\circ} \mathrm{C}$ until the analysis. Plasma $(120 \mu 1)$ was deproteinized by the addition of 30 $\mu 10.5 \mathrm{~N}$ hydrochloric acid, to which a $150 \mu 1$ internal standard (homoserine) was added. Protein was removed immediately by centrifuging at $8,000 \mathrm{rpm}$ for $30 \mathrm{~min}$. The deproteinized supernatant $(40 \mu 1)$ was transferred to a sampler vial, and 3-MH concentrations were analyzed by an automatic amino-acid analyzer (Hitachi 83550, Japan) using the standard physiological program described in the manual. A lithium-loaded ion-exchange column was used. The column effluent was mixed with o-phthalaldehyde (OPA) fluorescent derivatives of the primary amines. A standard mixture was run before and after each batch. No more than five samples were analyzed sequentially in a batch.

Statistical analysis. The data were expressed as M \pm SEM. The differences between the young, elderly and centenarians of each sex were evaluated by Duncan's multiple range test. A $p$ value of $<0.05$ was considered significantly different. 
Y.-C. CHAN et al.

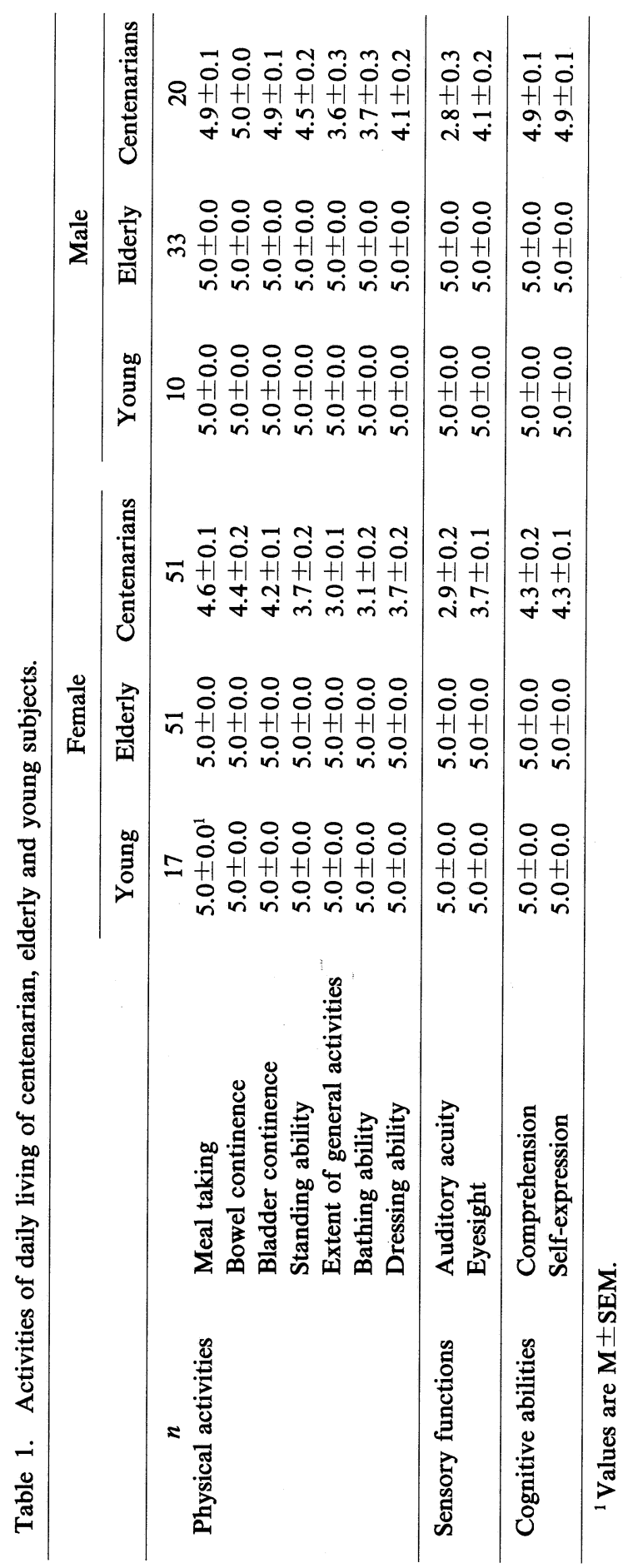

J. Nutr. Sci. Vitaminol. 


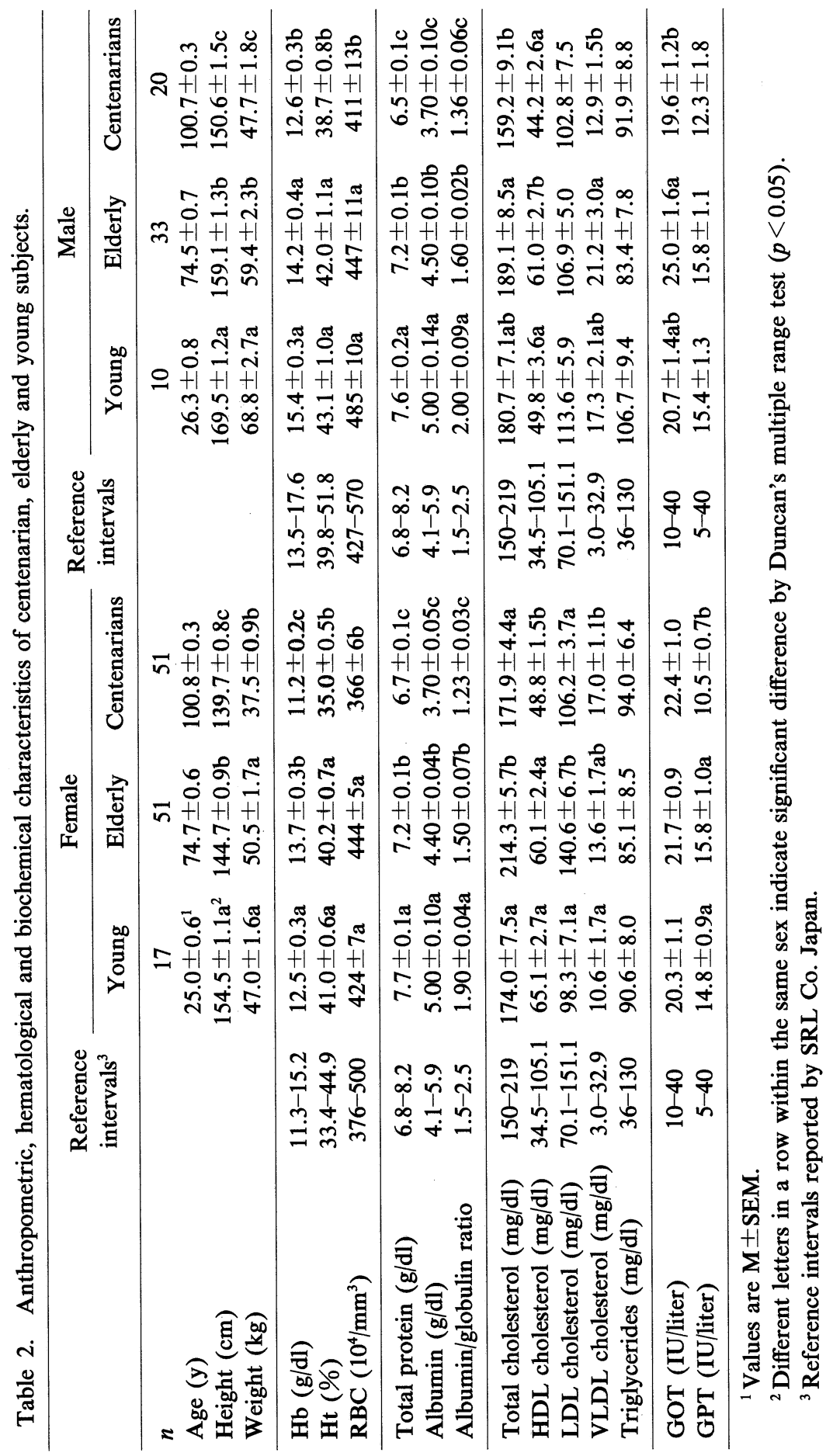



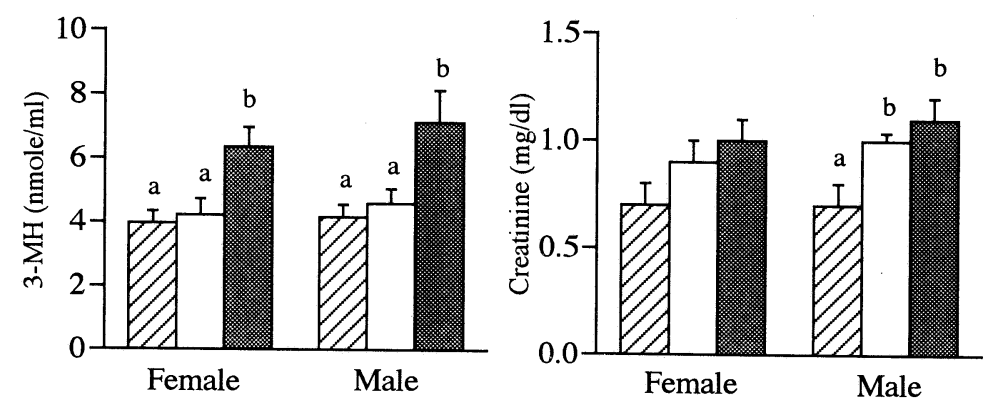

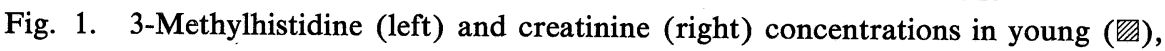
elderly ( $\square$ ) and centenarian (W) subjects. Values are $\mathbf{M} \pm$ SEM. Different letters within the same sex indicate significant difference by Duncan's multiple range test $(p<0.05)$.

\section{RESULTS}

Table 1 shows the ADL of the centenarian, elderly and young subjects. The elderly and young subjects had complete independence of physical activities, good sensory functions and cognitive abilities. The physical activities of the centenarians of both sexes were between the category of completely independent and independent but slow. The functions of auditory acuity and eyesight of the centenarians were poor but their cognitive abilities were still good.

Table 2 shows the mean age, body weight, height, hematological and biochemical values of the centenarian, elderly and young subjects. Reference intervals shown in the table were those reported by SRL Co., Japan, which are one of the most reliable values $(1,6)$. Most of the values except serum lipids, GOT and GPT of the centenarians were lower than those in the young subjects $(p<0.05)$; while the values for serum lipids were mostly similar between young and centenarian subjects. As compared to the young subjects, the elderly in their 70s had: 1) shorter height; 2) heavier weight in females and lighter weight in males; 3) lower total protein and albumin levels and albumin/globulin (A/G) ratios; and 4) higher totaland LDL-cholesterol levels in females and HDL-cholesterol levels in males $(p<$ 0.05). The GOT and GPT concentrations in all three groups were similar.

Figure 1 shows the concentrations of plasma 3-MH and serum creatinine. For both sexes, the plasma 3-MH levels of the centenarians were higher than those in the other two groups $(p<0.05)$, which had similar levels. Creatinine concentration increased with aging in both sexes, and was significantly higher in the elderly and centenarian subjects than in the young subjects in the male groups $(p<0.05)$.

\section{DISCUSSION}

ADL was an important index to characterize our volunteer subjects. The assessment of ADL was based on the index of Inoue's activity of daily living, which 
has been widely used for the aged in Japan (7). Our young and elderly subjects had high ADL, whereas the centenarians had low ADL; especially to the extent of general activities, bathing and dressing ability and sensory functions. However, none of the centenarians were bed ridden and all could ambulate, indicating that they were a rather healthy group of centenarians $(8)$.

Anthropometric measurements are most affected by the aging process. Aging was associated with decreased height and weight except the weight of elderly female subjects. The results showed that centenarians were very small and thin.

Hematological parameters are often used as part of a comprehensive nutritional assessment. The elderly are often anemic (9). Our subjects in their $70 \mathrm{~s}$ had rather similar values of $\mathrm{Hb}, \mathrm{Ht}$ and $\mathrm{RBC}$ as the young subjects. However, the centenarians had lower values than the other age groups $(p<0.05)$ and were near the lower reference limits. The results indicate that the drop in these values after about 70 years of age were sharp, which is supported by the reviewed values of a large number of Japanese up to $85 \mathrm{y}(1)$.

Serum total protein and albumin concentrations and $A / G$ ratio are fairly reliable measures for the assessment of nutritional status, and decreases in these parameters with aging are well known $(1,10)$. It was very similar in this study; the values decreased clearly with aging and were significantly different among the three age groups $(p<0.05)$. The values of the centenarians were lower than the lower reference limits. Albumin is produced in the liver and is reduced by major chronic diseases, particularly hepatic disease. However, GOT and GPT concentrations were similar among the three age groups, suggesting that the centenarians did not have any specific hepatic diseases and the low values may be due to reduced synthesis.

Nakamura (1) has summarized the age-related (from 20s to 80 s) changes of serum lipids from several institutes in Japan. Total cholesterol concentration increases until 50-70y old $(1,10)$ and then decreases as aging continues $(1,11)$. A similar tendency was observed in our subjects. The values of the centenarians were similar to the levels of the young subjects and were within the reference intervals (6). Lower serum cholesterol concentration is an effective way to reduce cardiovascular morbidity and mortality, but increases mortality in various diseases (12) and also induces immune dysfunction (13). Low serum cholesterol concentration is also associated with a high incidence of stroke in Japan, because the blood vessels of hypocholesterolemic patients are weak and easily broken by high blood pressure (14). The age-related change of HDL-cholesterol level is rather small (1). In this study, the concentrations of the centenarian groups were lower than those of the young groups but were within the reference intervals. The reduction of HDLcholesterol may accelerate the development of atherosclerosis by impairing the clearance of cholesterol from the arterial wall (15). A negative correlation between HDL-cholesterol and the development of coronary artery disease exists $(16,17)$. Since heart disease and stroke are the major causes of death in Japanese elderly (18), moderate concentrations of all the serum lipoprotein cholesterols found in our

Vol. 43, No. 1, 1997 
centenarian subjects must be important factors for their longevity.

The measurement of 3-MH has been recognized as a technique useful for assessing muscle protein breakdown $(3,4)$. The plasma concentration of 3-MH was significantly higher in the centenarians than in the other two age groups $(p<0.05)$, and was similar between the young and elderly subjects. However, creatinine concentration, which is an indicator of kidney function, was also high in the centenarians. Therefore, the high plasma 3-MH in the centenarians might not be due to accelerated muscle atrophy but rather due to poor kidney functions.

In summary, the centenarians had shrunken stature, low hematological values and poor protein nutrition but rather normal lipid nutrition. However, the elderly subjects in their 70 s could still maintain most nutritional parameters in relatively normal condition.

We are grateful to Mr. Masami Ogura for the analysis of 3-methylhistidine, to $\mathrm{Mr}$. Andrew A. Adjei for his help in the correction of English and to the staff of the Department of Nutrition and Research Center of Comprehensive Medicine, University of the Ryukyus, for their various cooperation in this research.

\section{REFERENCES}

1) Fujii, J., Kanazawa, Y., Nakamura, H., and Hata, Y. (1993): Roujin Rinsyo Kensati no Kangaekata-Kijun Han-i [Evaluation of Clinical Laboratory Data in the Elderly (Reference Intervals)], ed. by Kawai, H., Yakugyou Jihou-sya, Tokyo, pp. 1-69 (in Japanese).

2) Trankoff, S. P., and Norris, A. H. (1977): Effect of muscle mass decrease on age-related BMR changes. J. Appl. Physiol., 43, 1001-1006.

3) Young, V. R., and Munro, H. N. (1978): $N^{\tau}$-methylhistidine and muscle protein turnover: an overview. Fed. Proc., 37, 2291-2300.

4) Funabiki, R. (1990): Protein turnover and nutritional state, in Nutrition: Proteins and Amino Acids, ed. by Yoshida, A., Naito, H., Niiyama, Y., and Suzuki, T., Japan Sci. Soc. Press, Tokyo, pp. 35-48.

5) Nagasawa, T. (1996): Evaluation of muscle protein degradation by 3-methylhistidine concentration in blood. Hissu Amino-san Kenkyu Kaishi (Reports of the Research Committee of Essential Amino Acids), 145, 52-55 (in Japanese).

6) SRL Co. (1996): SRL Handbook of Clinical Examination, SRL Co., Tokyo pp. 104405.

7) Akisaka, M., Asato, L., Chan, Y. C., Suzuki, M., Uezato, T., and Yamamoto, S. (1996): Energy and nutrient intakes of Okinawan centenarians. J. Nutr. Sci. Vitaminol., 42, 241-248.

8) Suzuki, M., Akisaka, M., Ashitomi, I., Higa, K., and Nozaki, H. (1995): Chronological study concerning ADL among Okinawan centenarians. Jpn. J. Geriat., 32, 416423 (in Japanese).

9) Howe, R. B. (1983): Anemia in the elderly. Common causes and suggested diagnostic approach. Postgrad. Med., 73, 153-160.

10) Fulop, T., Worum, I., Varga, P., Foris, G., Bars, L., Mudri, K., and Leovey, A. 
(1989): Blood laboratory parameters of carefully selected healthy elderly people. Arch. Gerontol. Geriatr., 8, 151-163.

11) Nakamura, H. (1994): Desirable values of plasma lipids in the aged. Jpn. J. Geriat., 31, 275-278 (in Japanese).

12) Muldoon, M. F., Manuck, K. A., and Matthews, K. A. (1990): Lowering cholesterol concentrations and mortality: a quantitative review of primary prevention trails. $B r$. Med. J., 301, 309-314.

13) Shor-Posner, G., Basit, A., Lu, Y., Cabrejos, C., Chang, J., Fletcher, M., ManteroAtienza, E., and Baum, M. K. (1993): Hypocholesterolemia is associated with immune dysfunction in early human immunodeficiency virus-1 infection. Am. J. Med., 94, 515-519.

14) Komachi, Y., Iida, M., Shimamoto, T., Chikayama, Y., and Takahashi, H. (1971): Geographic and occupational comparisons of risk factors in cardiovascular disease in Japan. Jpn. Circ. J., 35, 189-207.

15) Miller, N. E. (1987): Associations of high-density lipoprotein subclasses and lipoproteins with ischemic heart disease and coronary atherosclerosis. Am. Heart. J., 113, 589-597.

16) Greens, M. S., Heiss, G., Rifkind, B. M., Cooper, G. R., Williams, O. D., and Tyroler, H. A. (1985): The ratio of plasma high-density lipoprotein cholesterol to total and low-density lipoprotein cholesterol: age-related changes and race and sex differences in selected North American populations. The Lipid Research Clinics Program Prevalence Study. Circulation, 72, 93-104.

17) Miller, N. E., Harmmett, F., Saltissi, S., Rao, S., van-Zeller, H., Coltart, J., and Lewis, B. (1981): Relation of angiographically defined coronary artery disease to plasma lipoprotein subfractions and apolipoproteins. Br. Med. J. Clin. Res. Ed., 282, 17411744.

18) Statistics and Information Department Minister's Secretariat Ministry of Health and Welfare (1994): Health and Welfare Statistics in Japan, Health and Welfare Statistics Association, Tokyo, pp. 43-50. 